\title{
Diagnosis of important fixed coronary stenosis in patients with variant angina by exercise tests after treatment with calcium antagonists
}

\author{
HARUO ARAKI, NOBU HAYATA, TAKEYUKI MATSUGUCHI, \\ AKIRA TAKESHITA, MOTOOMI NAKAMURA \\ From the Research Institute of Angiocardiology and Cardiovascular Clinic, Faculty of Medicine, Kyushu \\ University, Fukuoka, fapan
}

SUMMARY A 12 lead electrocardiogram was recorded during treadmill exercise in 57 patients with variant angina in whom coronary angiography was performed. Thirty six patients performed exercise tests with and without calcium antagonists, and 21 performed them only with calcium antagonists. In 55 patients calcium antagonists had prevented spontaneous attacks of variant angina for more than two days before the test. The other two patients were given a single dose of diltiazem $(90 \mathrm{mg})$ two hours before the test. Exercise testing without calcium antagonists induced ST segment elevation with chest pain in nine patients, ST segment depression in 10 (nine with chest pain), and no important shift of the ST segment in 17. Five patients had severe coronary stenosis $(\geqslant 75 \%)$ and all of them showed positive response. Thirty one patients had no important coronary stenosis and 14 of them showed positive response. The sensitivity of the exercise test in detecting a coronary stenosis $\geqslant 75 \%$ was $100 \%$ without calcium antagonists but the specificity was low $(55 \%)$. When the exercise test was done in patients taking calcium antagonists, only two (specificity $96 \%$ ) of 48 patients without severe coronary stenosis showed positive response (elevation of ST segment in one and depression in another) whereas all nine patients with severe coronary stenosis had a positive response (depression of ST segment in six and elevation in three (sensitivity $100 \%$ ).

It is concluded that exercise testing with calcium antagonists may be a useful method for detecting severe coronary stenosis in patients with variant angina.

Coronary lesions in patients with effort angina are reliably detected by exercise induced shifts in the ST segment. ${ }^{12}$ In variant angina, however, exercise causes coronary spasm in some patients and hence elevation or depression of the ST segment even in the absence of an important coronary lesion. ${ }^{3-9}$ For this reason exercise testing may not be helpful in the evaluation of coronary lesions in patients with variant angina. Reliable exercise testing depends on the complete prevention of coronary spasm during exer-

Requests for reprints to Dr Haruo Araki, Research Institute of Angiocardiology and Cardiovascular Clinic, Faculty of Medicine, Kyushu University, 3-1-1 Maidashi, Higashi-ku, Fukuoka, 812 Japan.

Accepted for publication 3 March 1986 cise. Calcium antagonists prevent spontaneous and exercise induced coronary spasm. ${ }^{710}$ We have therefore evaluated the diagnostic value of exercise tests in variant angina in patients taking calcium antagonists and compared the result with that obtained when exercise testing was performed without calcium antagonists.

\section{Patients and methods}

PATIENT SELECTION

We studied patients with variant angina admitted to Kyushu University Hospital between 1976 and 1984. Variant angina was diagnosed when the patient showed transient ST segment elevation of $\geqslant 1 \mathrm{~mm}$ on a 12 lead electrocardiogram during chest pain at rest with no subsequent rise in serum enzyme 
concentrations. All 57 patients were assessed by both treadmill exercise test and coronary angiography. Thirty six patients underwent exercise tests with and without calcium antagonists and the remaining 21 only performed the test after taking calcium antagonists. Forty six were male and 11 were female (age range 31 to 70 years, mean (SD) 55 (9)). One patient had a history of previous myocardial infarction. All were in sinus rhythm and showed no arrhythmias before the exercise test.

All patients underwent coronary angiography by Judkins' or Sones' techniques. Multiple views of each vessel were filmed. When a lesion was noted, the vessel was filmed again in multiple views after administration of sublingual or intravenous glyceryl trinitrate. The coronary artery diameter was measured on the projected screen by means of slide callipers. Coronary stenosis was expressed as the reduction in diameter at the stenosis in relation to the mean diameter of two non-stenotic portions measured just proximal and distal to the stenosis. Patients were divided into three groups according to the degree of coronary stenosis. Group 1 (minimal) consisted of patients with normal coronary arteries or with stenosis of $<50 \%$. Group 2 (moderate) consisted of patients with coronary stenosis of $50 \%-74 \%$, and group 3 (severe) had coronary stenosis $\geqslant 75 \%$.

\section{TREADMILL EXERCISE TEST}

The treadmill exercise test was performed according to a Bruce or modified Bruce protocol. Exercise tests were performed between 7 am and $10 \mathrm{am}$. Medication with cardiovascular activity was withheld for at least two days before the test. An exercise test was performed one to three hours after oral administration of the calcium antagonist and no more than two weeks before coronary angiography; the interval between the two exercise tests ranged from two days to two weeks. Blood pressure and heart rate were measured every minute during exercise and the recovery period of 6-15 min. Three leads of a selected electrocardiogram were continuously monitored during exercise. A 12 lead electrocardiogram was recorded before the exercise and once a minute during the recovery period until the electrocardiogram reverted to normal, and also during chest pain. The end point of the exercise test was reached when (a) the submaximal heart rate (more than $85 \%$ of age matched maximal heart rate) was reached, (b) chest pain developed, (c) the ST segment changed by $\geqslant 2 \mathrm{~mm}$, (d) exhaustion or leg fatigue developed, or (e) systolic blood pressure fell by $\geqslant 20 \mathrm{~mm} \mathrm{Hg}$. All patients reached the target heart rate or had chest pain during the exercise test.

ST segment changes were deemed to be positive when horizontal or down sloping depression of $\geqslant 1$ mm occurred or when ST segment elevation of $\geqslant 1$ $\mathrm{mm}$ was seen. Informed consent was obtained from each patient before the exercise test and coronary angiography.

ADMINISTRATION OF CALCIUM ANTAGONISTS Treadmill tests were performed 1-3 hours after oral administration of calcium antagonists-120 to 360 $\mathrm{mg} /$ day of diltiazem in 50 patients, 40 to $80 \mathrm{mg} /$ day of nifedipine in two, $160 \mathrm{mg} /$ day of nicardipine in two, and a combination of diltiazem $240 \mathrm{mg}$ and nifedipine $40 \mathrm{mg} /$ day as three or four divided doses in one patient. These doses abolished the rest angina as well as the painless ST segment elevation, which was confirmed by Holter electrocardiographic monitoring. ${ }^{11}$ Calcium antagonists were given for $\geqslant 2$ days before the exercise test. Ten of these patients were given $90 \mathrm{mg}$ of diltiazem two hours before the test in order to achieve plasma concentrations in the therapeutic range. ${ }^{12}$ Two patients had only one dose of diltiazem $(90 \mathrm{mg})$ two hours before the treadmill test. Exercise tests were not performed for two hours after a meal. The 12 patients who were given an additional or single dose of $90 \mathrm{mg}$ diltiazem were studied prospectively. The others were studied retrospectively.

\section{STATISTICAL ANALYSIS}

The diagnostic values of exercise tests with and without a calcium antagonist were compared by a $\chi^{2}$ test with Yates's correction. The prevalence of positive response to exercise tests in the three groups of patients with different angiographic findings was analysed by the variance test for homogeneity of the binominal distribution. ${ }^{13}$ The pressure-rate product of exercise tests with and without calcium antagonist was compared by Student's $t$ test for paired data. The pressure-rate products in the three groups with different angiographic findings were compared by analysis of variance. $P$ values of $<0.05$ were considered to be statistically significant.

\section{Results}

Table 1 shows the clinical features of patients and their angiographic findings. Thirty six patients had normal coronary arteries or mild coronary stenosis (group 1) and 12 patients had moderate coronary stenosis (group 2). Nine had severe coronary stenosis (group 3); seven of them had single vessel disease and two had two vessel disease. Before admission 28 patients had only rest angina and 29 had both rest and effort angina. Most patients with severe coronary stenosis had both rest and effort angina. ST segment elevation that was typical of variant angina 
Table 1 Patient profiles

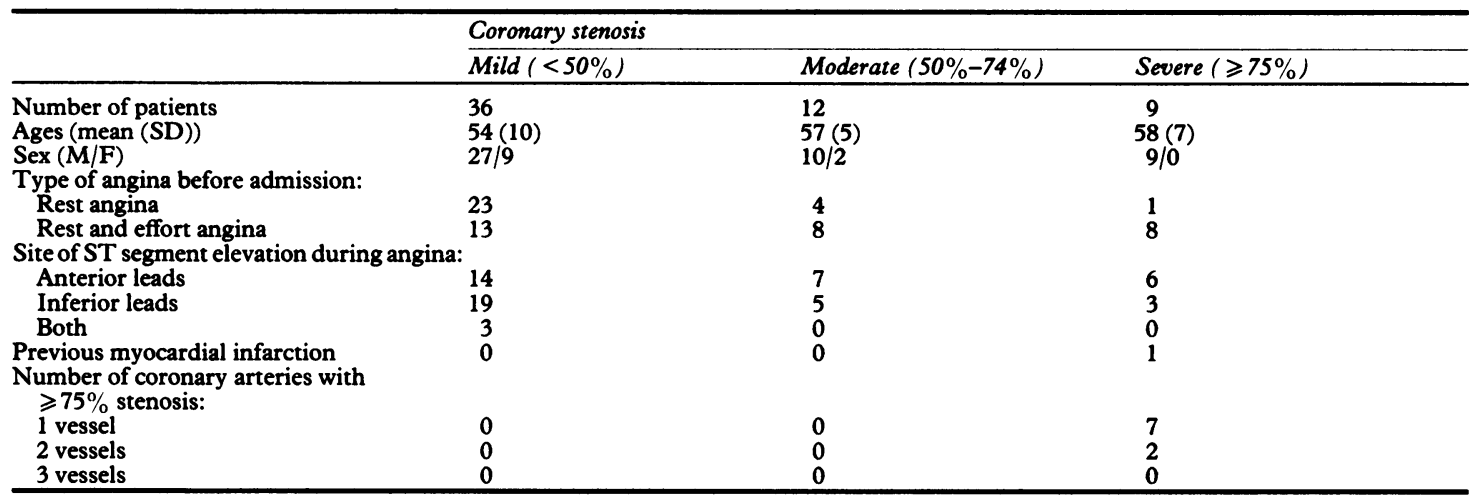

was seen in anterior leads in 27 patients, in inferior leads in 27, and in both in three.

Figures 1 and 2 show 12 lead electrocardiograms during treadmill exercise in a patient without and with calcium antagonists. Without calcium antagonists he complained of chest pain after 5 min of exercise on Bruce's protocol and transient ST segment elevation developed in leads II, III, and aVF (Fig. 1). A repeat exercise test also provoked chest pain with ST segment elevation. When an exercise test
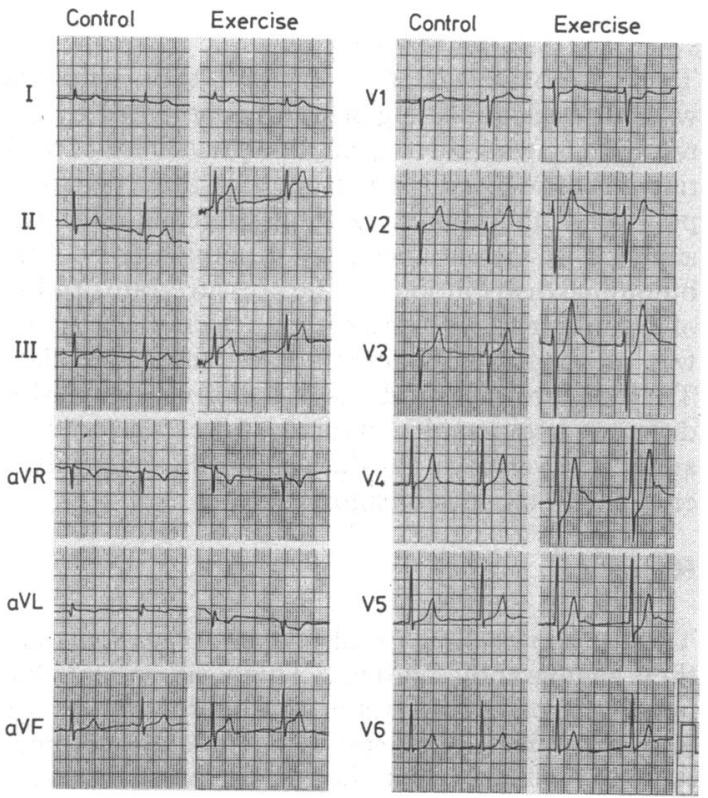

Fig. 1 Twelve lead electrocardiograms in a patient at rest and during chest pain after exercise. The exercise test was done without calcium antagonists. Chest pain was associated with the development of ST segment elevation in leads $I I$, $I I I$, and $a V F$; and reciprocal $S T$ segment depression in leads $a V L$ and VI-VS. was performed after he had taken a calcium antagonist, he could exercise for up to $9 \mathrm{~min}$ without chest pain or ST segment change (Fig.2). Coronary angiography showed only a mild stenosis $(25 \%)$ in the left anterior descending coronary artery.

Figures 3 and 4 show 12 lead electrocardiograms in another representative patient. During exercise

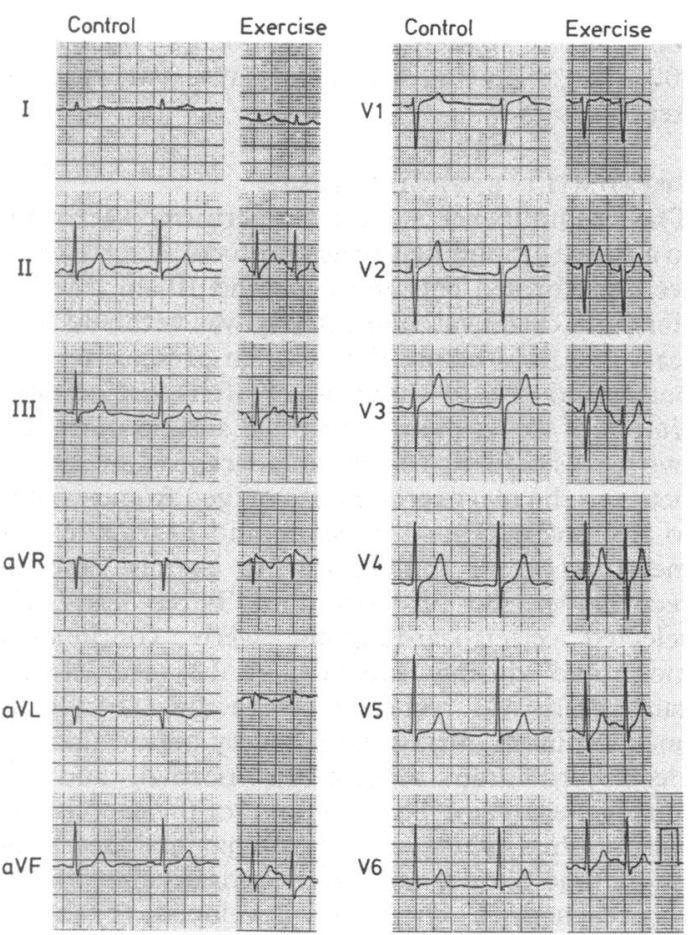

Fig. 2 Treadmill exercise test in the same patient as Fig. 1 while he was on a calcium antagonist. He attained age matched maximal heart rate without chest pain or $S T$ segment shifts. Coronary angiography showed no important coronary stenosis in this patient. 

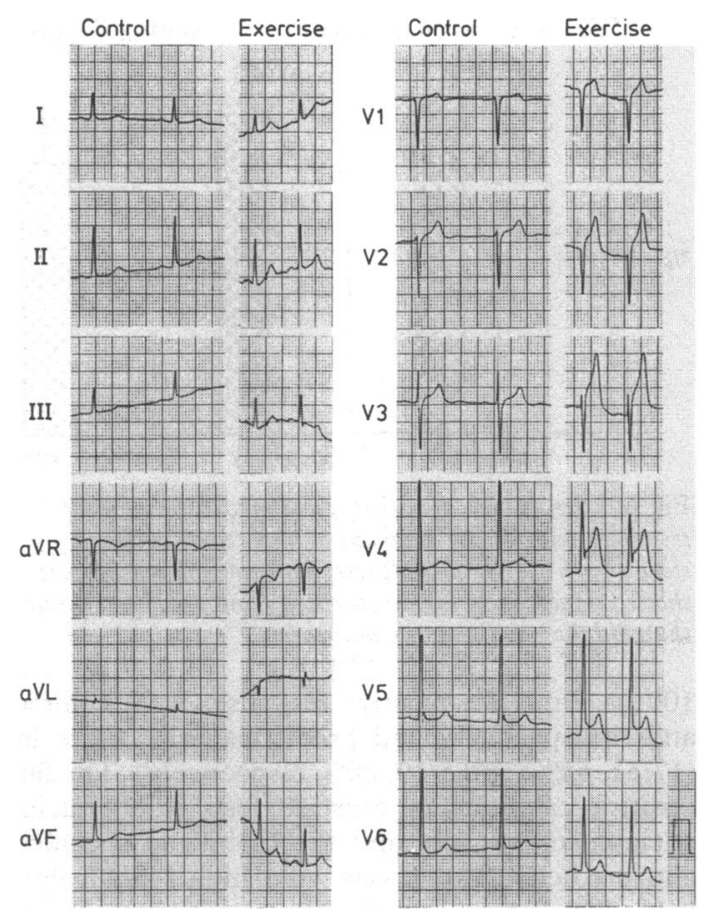

Fig. 3 Twelve lead electrocardiograms in a patient at rest and after exercise without calcium antagonists. An anginal attack developed with ST segment elevation in leads V1-V5.

tests performed without calcium antagonists he complained of chest pain after $3 \mathrm{~min}$ on the Bruce protocol and ST segment elevation developed in leads V1 to V5 (Fig. 3). Unlike the patient in Figs. 1 and 2 he had chest pain on exercise even after calcium antagonist had been given; ST segment depression developed in leads V4 to V6 (Fig. 4). Coronary angiography showed a $90 \%$ stenosis of the left anterior descending coronary artery.

\section{EXERCISE TESTS WITH AND WITHOUT \\ CALCIUM ANTAGONIST}

Thirty six patients performed exercise tests without calcium antagonists; all of them had spontaneous attacks of angina that occurred a few times a month to several times a day. Nineteen of 36 patients responded positively to the exercise test. Nine of them had ST segment elevation and 10 had ST segment depression. Chest pain developed during 18 positive tests. Figure 5 shows the prevalence of positive response in the three groups of patients with different degrees of organic coronary stenosis. All five patients with severe coronary stenosis developed important ST segment shifts with chest pain and seven of the eight patients with moderate coronary stenosis had a positive response. Seven $(30 \%)$ of the 23 patients with normal coronary angiogram or mild coronary stenosis also showed a positive response, but this was significantly less common than the other two patient groups $(p<0.025)$.

Figure 6 shows the results of 57 exercise tests after calcium antagonists. All nine patients with severe coronary stenosis showed a positive response. But 11 of the 12 patients with moderate coronary stenosis did not develop significant shifts of ST segment or chest pain. Only one of the 36 patients with normal coronary angiogram or mild coronary stenosis responded positively. Positive responses were significantly more common in patients with severe coronary stenosis than in the other two patient groups $(p<0.005)$. Of the 11 patients with a positive response, four had ST segment elevation and seven had ST segment depression (Fig. 6). Nine patients had chest pain during the exercise test.

Figure 7 gives the sensitivity, specificity, and predictive value of the exercise test with and without calcium antagonists when it was used to diagnose severe coronary stenosis. The sensitivity of exercise tests without calcium antagonists was high $(5 / 5$,

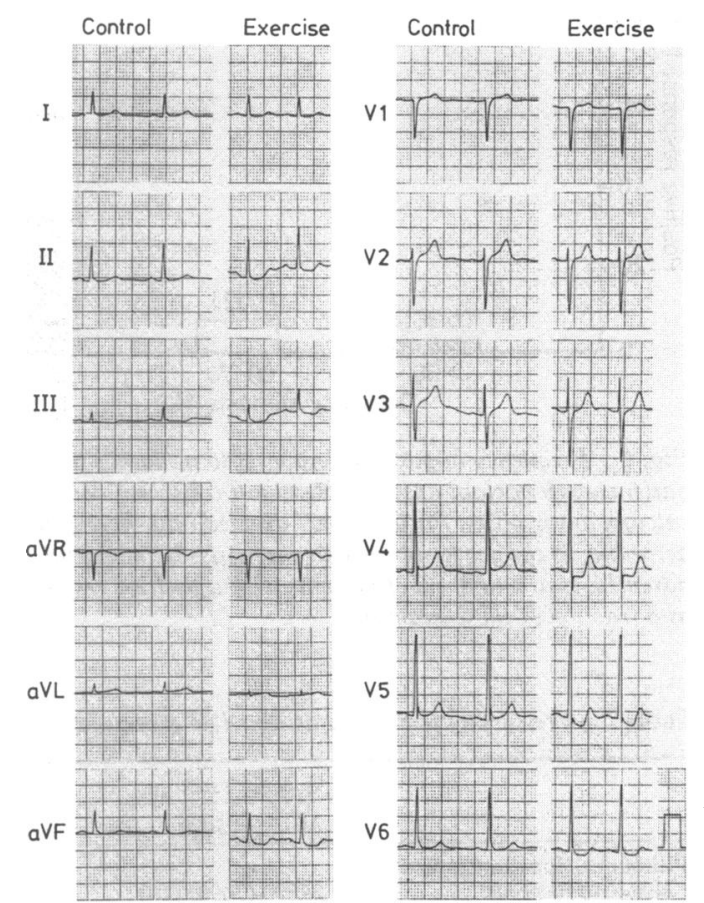

Fig. 4 Treadmill exercise test in the same patient as Fig. 3. Chest pain developed even with calcium antagonists, and $S T$ segment depression was seen in leads V4-V6. He had severe coronary stenosis (90\%) of the left anterior descending coronary artery. 


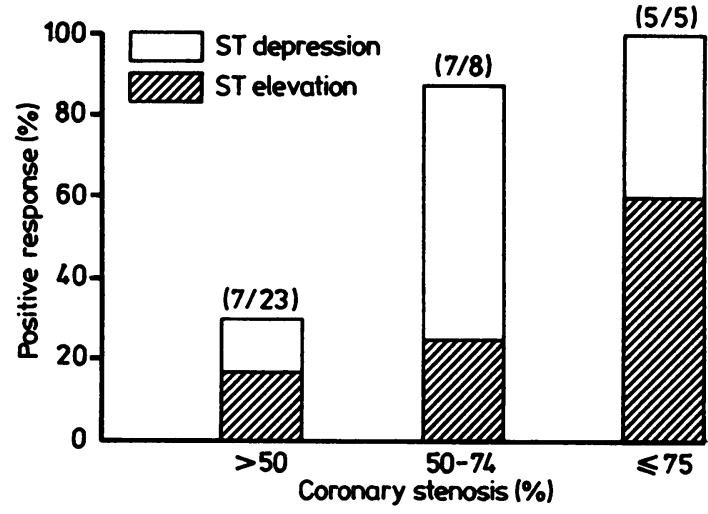

Fig. 5 Frequency of positive responses to treadmill tests in patients with variant angina. Treadmill tests were done without calcium antagonists. Patients were divided into three groups according to the degree of coronary stenosis. Numbers in parentheses are numbers of patients with a positive response as a fraction of those examined.

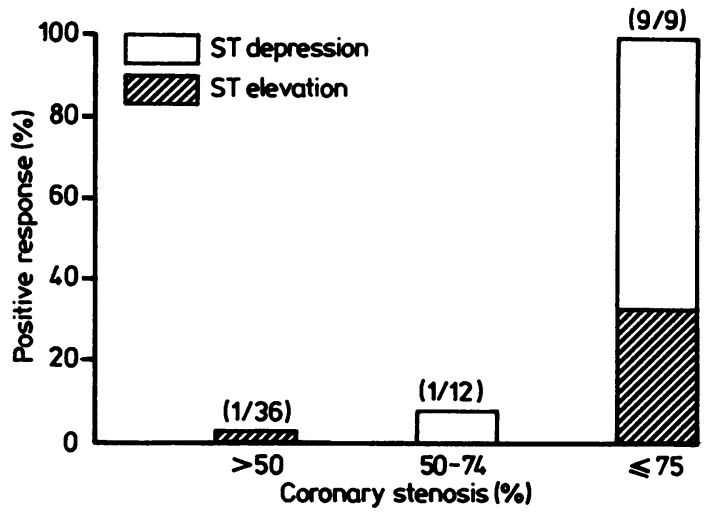

Fig. 6 Frequency of positive responses to treadmill tests in patients with variant angina. Treadmill tests were done with calcium antagonists. Patients were divided into three groups according to the degree of coronary stenosis. Numbers in parentheses are numbers of patients with a positive response as a fraction of those examined.

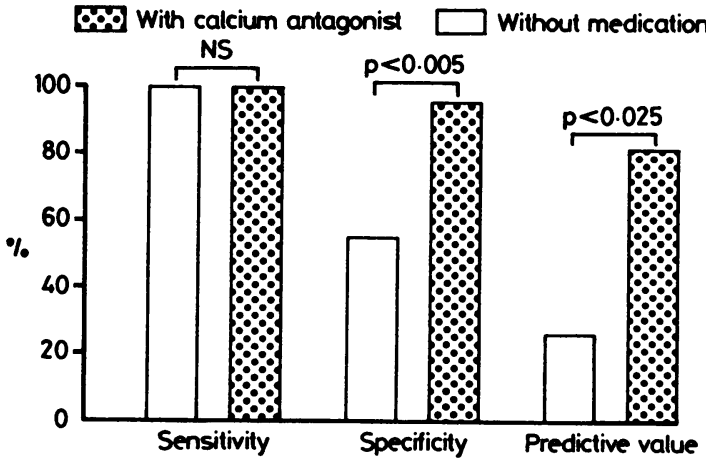

Fig. 7 Sensitivity, specificity, and predictive value of treadmill tests for the diagnosis of important coronary stenosis (75\%). Treadmill tests with calcium antagonists showed significantly higher specificity and predictive value than did those without any medication.

$100 \%$ ). But false positive responses were common and the specificity and predictive value were low $(17 / 31,55 \%$; and $5 / 19,26 \%$ respectively). The false positive rate during exercise tests after calcium antagonists was low and the calculated specificity and predictive value were very high $(46 / 48,96 \%$; $9 / 11,82 \%$ respectively). The sensitivity was also high (9/9).

\section{PRESSURE-RATE PRODUCT ATTAINED ON EXERCISE TESTING}

Table 2 gives the pressure-rate product attained on treadmill tests in three different patient groups. The pressure-rate product was significantly lower in patients with a positive response than in those with a negative response. This was found in exercise tests both with and without calcium antagonists. In exercise testing without calcium antagonists patients with severe coronary stenosis tended to develop angina and/or ischaemic ST segment changes at a lower pressure-rate product $(14700(5000))$; but this difference was not statistically significant. In patients with a negative exercise response the administration of calcium antagonist did not change the pressure-rate product.

Table 2 Pressure-rate product attained on exercise tests with and without calcium antagonists

\begin{tabular}{lllll}
\hline & Coronary stenosis & & & Total \\
\cline { 2 - 4 } & Mild $(<50 \%)$ & Moderate $(50 \%-74 \%)$ & Severe $(\geqslant 75 \%)$ & \\
\hline Without calcium antagonist: & $24800(7090)(16)$ & $33800(1)$ & $-(0)$ & $25300(7200)(17)$ \\
Negative response & $19900(5190)(7)$ & $19500(3450)(7)$ & $14700(5000)(5)$ & $18400(4870) \star(19)$ \\
Positive response & $26000(4950)(35)$ & $25800(2470)(11)$ & $-(0)$ & $26000(4450)(46)$ \\
With calcium antagonist: & $16700(1)$ & $24800(1)$ & $17800(3820)(9)$ & $18400(4040) \star(11)$ \\
Negative response & Positive response & &
\end{tabular}

Values are mean (SD). Italic numbers in parentheses are number of patients.

${ }^{\star} \mathrm{p}<0.01$, compared with negative response group. 


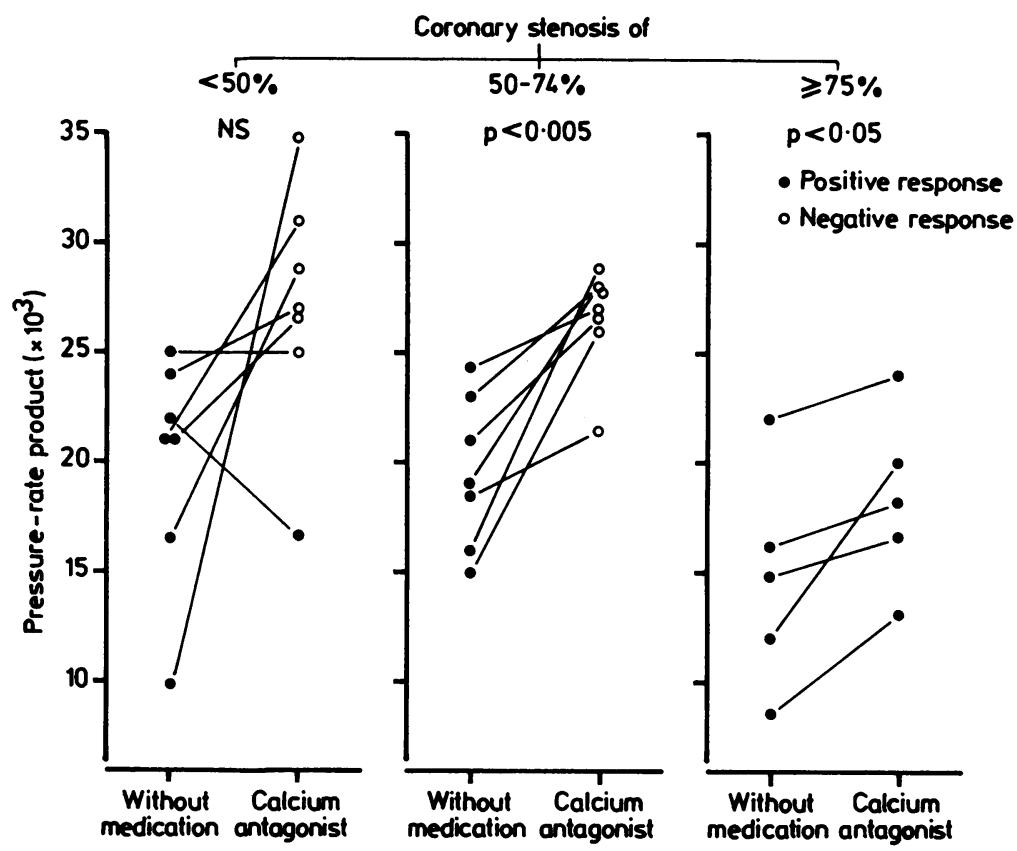

Fig. 8 Pressure-rate product in patients who responded positively to the exercise test without calcium antagonist and the change after calcium antagonists had been given. Closed circles indicate a positive response and open circles a negative response. In most patients without important coronary stenosis $(\geqslant 75 \%)$ the exercise response changed from positive to negative after calcium antagonist and a higher pressure-rate product was attained.

Nineteen patients showed a positive response to exercise testing without calcium antagonists. Figure 8 shows the pressure-rate product of each patient as well as changes in the product after calcium antagonists. With calcium antagonists the exercise tests resulted in a negative response in 13 patients and the pressure-rate product increased in 12 of these patients. One patient without severe coronary stenosis responded positively to the exercise test even with calcium antagonist and angina with ST segment elevation developed at an even lower pressurerate product. The pressure-rate product in five patients with severe coronary stenosis increased slightly after calcium antagonists but remained low compared with that in other patients $(p<0.05 v \mathrm{vs}$ mild coronary stenosis, and $\mathrm{p}<0.01$ vs moderate coronary stenosis).

\section{Discussion}

Variant angina can be provoked by hyperventilation, ${ }^{14}$ alcohol, ${ }^{15-17}$ and intravenous administration of ergometrine ${ }^{18-21}$ or histamine. ${ }^{22}$ Several studies have also indicated that exercise can provoke an anginal attack in some patients with variant angina. ${ }^{3-8}$ In other words some patients with variant angina develop chest pain with ischaemic ST segment shifts in the absence of severe coronary stenosis. For this reason the exercise test, which gives very useful information for the evaluation of coronary lesions in effort angina, ${ }^{12}$ seems to be of limited value in patients with variant angina. In the present study 14 of 31 patients without severe coronary stenosis developed angina or ST segment changes or both during treadmill exercise tests. This result is compatible with that reported by Waters et al. In their study important coronary stenosis was defined as narrowing of $\geqslant 70 \%$ and 19 of 41 patients without coronary stenoses developed important ST segment shifts on exercise. ${ }^{7}$ Fukami et al reported a similar result. ${ }^{23}$ Thus the specificity of exercise tests for the diagnosis of important coronary lesions is low in patients with variant angina.

We found that patients with a coronary stenosis of $\geqslant 75 \%$ responded positively to the exercise test without calcium antagonists (sensitivity $100 \%$ ). In contrast to this Waters et al $^{7}$ found that 14 of 36 patients with coronary stenosis of $\geqslant 70 \%$ did not 
develop ST segment shifts on exercise testing. They speculated that an inadequate workload was the cause of this large proportion of negative results. It is probable that even in the absence of coronary spasm, ischaemic symptoms or signs will always develop in cases of severe fixed stenosis $(\geqslant 75 \%)$ if the patient can complete enough exercise.

Seven of 23 patients with a normal coronary angiogram or mild stenosis developed ST segment changes on exercise. In contrast, seven of eight patients with a moderate coronary stenosis showed a positive response, as did all five patients with severe coronary stenosis. The proportion of patients with positive responses was significantly higher in those with moderate or severe coronary stenoses $(12 / 13$, $92 \%)$ than in the remainder $(7 / 23,30 \%, \mathrm{p}<0.005)$. These results suggest that angina or ST segment shifts develop more easily in patients with moderate coronary stenosis $(50-74 \%)$ than in those with mild stenoses or none. Larger studies would be needed to confirm this.

Calcium antagonists prevent coronary spasm, and thus pain, in patients with variant angina. ${ }^{24-27}$ They also inhibit exercise induced coronary spasm. ${ }^{7-9}$ A patient taking a calcium antagonist would be expected to have a positive response to exercise only when important coronary stenoses are present, since the false positive response caused by coronary spasm would be abolished.

The results of exercise tests in 57 patients taking calcium antagonists gave a sensitivity of $100 \%$ and a specificity of $96 \%$ for the diagnosis of coronary stenosis of $\geqslant 75 \%$. Most patients with coronary stenoses of $50-74 \%$, however, had a negative response to the exercise test and the sensitivity of the test was only $48 \%$. An exercise test performed by a patient taking a calcium antagonist may be helpful in the detection of severe coronary stenoses which cause myocardial ischaemia as a result of an increased myocardial oxygen demand, but it cannot detect a moderate coronary stenosis which may cause other coronary events such as thrombotic occlusion.

The specificity of the test will be increased and the false positive response rate reduced by inhibition of the spasm before the exercise test. Spontaneous attacks of variant angina were abolished by calcium antagonists in 55 of 57 patients before the exercise test. Holter monitoring of these patients also confirmed the disappearance of painless ST segment elevation.

ST segment elevation with chest pain developed during tests in four patients taking calcium antagonists. One case had a coronary stenosis of $<50 \%$. It seems most likely that exercise induced the coronary spasm in this patient despite treatment with a cal- cium antagonist. In the other three patients, who had coronary stenosis of $\geqslant 75 \%$, ST segment elevation may also be an indication of the exercise induced coronary spasm. We do not know whether a higher dose of calcium antagonist would have abolished ST segment elevation in these patients. Exercise can provoke ST segment elevation with chest pain even when doses of calcium antagonists sufficient to abolish the spontaneous attack of variant angina are being taken.

Two patients had one $90 \mathrm{mg}$ dose of diltiazem two hours before the exercise test and we cannot confirm that in these patients the spontaneous attacks were abolished. Both patients showed a true negative response. This single dose was high, but we saw no complications in these two patients or in the 10 patients who were given $90 \mathrm{mg}$ of diltiazem before the test in addition to their daily doses. It would be useful to know whether the administration of a single large dose of calcium antagonist is sufficient to inhibit exercise induced coronary spasm.

Calcium antagonists reduce blood pressure and heart rate to various degrees, and thus they may decrease the pressure-rate product. $A$ decrease in the pressure-rate product by calcium antagonist could have been responsible for the negative response to exercise tests. But the pressure-rate product increased significantly after calcium antagonists in those patients whose positive response to exercise became negative after calcium antagonists. This suggests that calcium antagonists improve the response to exercise not solely through reduction of myocardial oxygen consumption, but also through an improvement in oxygen supply either by inhibition of coronary spasm or by some other mechanism. In patients with severe coronary stenosis, however, the pressure-rate product remained low even after administration of a calcium antagonist. This indicates that in such patients doses of these antagonists that are large enough to inhibit spontaneous attacks of angina do not improve myocardial oxygen supply during exercise.

\section{References}

1 Goldschlager N, Selzer A, Cohn K. Treadmill stress tests as indicators of presence and severity of coronary artery disease. Ann Intern Med 1976;85:277-86.

2 McNeer JF, Margolis JR, Lee KL, et al. The role of the exercise test in the evaluation of patients for ischemic heart disease. Circulation 1978;57:64-70.

3 Waters DD, Chaitman BR, Dupras G, Theroux P, Mizgala HF. Coronary artery spasm during exercise in patients with variant angina. Circulation 1979;59:580-5. 
4 Yasue H, Omote S, Takizawa A, Nagao M, Miwa K, Tanaka S. Circadian variation of exercise capacity in patients with Prinzmetal's variant angina: role of exercise-induced coronary arterial spasm. Circulation 1979;59:938-48.

5 Specchia G, De Servi S, Falcone C, et al. Coronary arterial spasm as a cause of exercise-induced ST-segment elevation in patients with variant angina. Circulation 1979;59:948-54.

6 De Servi S, Falcone C, Gavazzi A, et al. The exercise test in variant angina: results in 114 patients. Circulation 1981;64:684-8.

7 Waters DD, Szlachcic J, Bourassa MG, Scholl JM, Theroux $P$. Exercise testing in patients with variant angina: results, correlation with clinical and angiographic features and prognostic significance. Circulation 1982;65:265-74.

8 Specchia G, De Servi S, Falcone C, et al. Significance of exercise-induced ST-segment elevation in patients without myocardial infarction. Circulation 1981; 63:46-53.

9 Bott-Silverman C, Heupler FA Jr, Yiannikas J. Variant angina: comparison of patients with and without fixed severe coronary artery disease. Am $\mathcal{F}$ Cardiol 1984;54:1173-5.

10 Freedman B, Dunn RF, Richmond DR, Kelly D. Coronary artery spasm during exercise: treatment with verapamil. Circulation $1981 ; 64: 68-75$.

11 Araki H, Koiwaya Y, Nakagaki O, Nakamura N. Diurnal distribuition of ST-segment elevation and related arrhythmias in patients with variant angina: study by ambulatory ECG monitoring. Circulation 1983;67:995-1000.

12 Koiwaya Y, Ashihara T, Nakamura M, Etoh A. Plasma concentration of diltiazem after oral administration in normal volunteers. Clin Ther 1981;3:436-40.

13 Snedecor GW, Cochran WG. Statistical methods. 6th ed. Iowa: The Iowa State University Press, 1967:240.

14 Yasue H, Nagao M, Omote S, Takizawa A, Miwa K, Tanaka S. Coronary arterial spasm and Prinzmetal's variant form of angina induced by hyperventilation and Tris-buffer infusion. Circulation 1978;58:56-62.

15 Fernandez D, Rosenthal J, Cohen L, Hammond G,
Wolfson S. Alcohol-induced Prinzmetal variant angina. Am $\mathcal{f}$ Cardiol 1973;32:238-9.

16 Takizawa A, Yasue H, Omote $S$, et al. Variant angina induced by alcohol ingestion. Am Heart $\mathcal{F} 1984$; 107:25-7.

17 Matsuguchi T, Araki H, Anan T, et al. Provocation of variant angina by alcohol ingestion. Eur Heart $\mathcal{f}$ 1984;5:906-12.

18 Schroeder JS, Bolen JL, Quint RA, et al. Provocation of coronary spasm with ergonovine maleate. Am $\mathcal{f}$ Cardiol 1977;40:487-91.

19 Curry RC, Pepsine CJ, Sabom BM, Feldman RL, Christie LG, Conti CR. Effects of ergonovine in patients with and without coronary artery disease. Circulation 1977;56:803-9.

20 Heupler FA Jr, Proudfit WL, Razavi M, Shirey EK, Greenstreet R, Sheldon WC. Ergonovine maleate provocative test for coronary arterial spasm. $A m \mathcal{F}$ Cardiol 1978;41:631-40.

21 Bertrand ME, LaBlanche JM, Tilmant PY, et al. Frequency of provoked coronary arterial spasm in 1089 consecutive patients undergoing coronary arteriography. Circulation 1982;65:1299-306.

22 Ginsburg R, Bristow MR, Kantrowitz N, Baim DS, Harrison DC. Histamine provocation of clinical coronary artery spasm: implications concerning pathogenesis of variant angina pectoris. Am Heart $\mathcal{f}$ 1981;102:819-22.

23 Fukami K, Tanaka N, Honda T, et al. The role of the exercise test in angina pectoris associated with coronary arterial spasm. Kokyu To funkan 1981; 29:1213-9. (In Japanese;abstract in English.)

24 Muller JE, Gunther SJ. Nifedipine therapy for Prinzmetal's angina. Circulation 1978;57:137-9.

25 Solberg LE, Nissen RG, Vlietstra RE, Callahan JA. Prinzmetal's variant angina-response to verapamil. Mayo Clin Proc 1978;53:256-9.

26 Kimura E, Kishida $H$. Treatment of variant angina with drugs: a survey of 11 cardiology institutes in Japan. Circulation 1981;63:844-8.

27 Schroeder JS, Lamb IH, Bristow MR, Ginsburg R, Hung J, McAuley BJ. Prevention of cardiovascular events in variant angina by long-term diltiazem therapy. $f$ Am Coll Cardiol 1983;1:1507-11. 